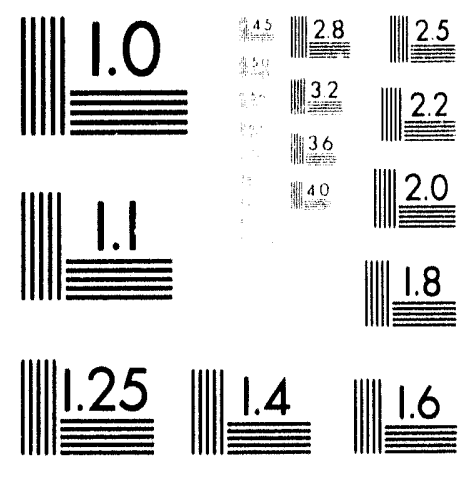



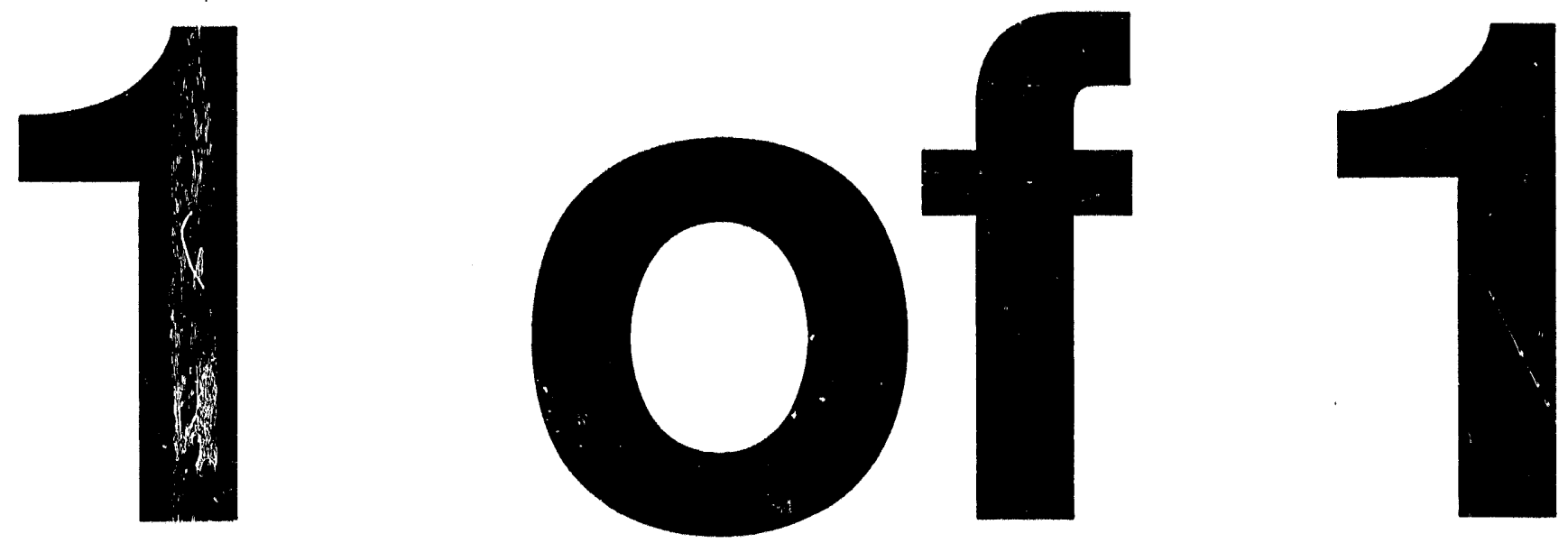

$\nabla$ 


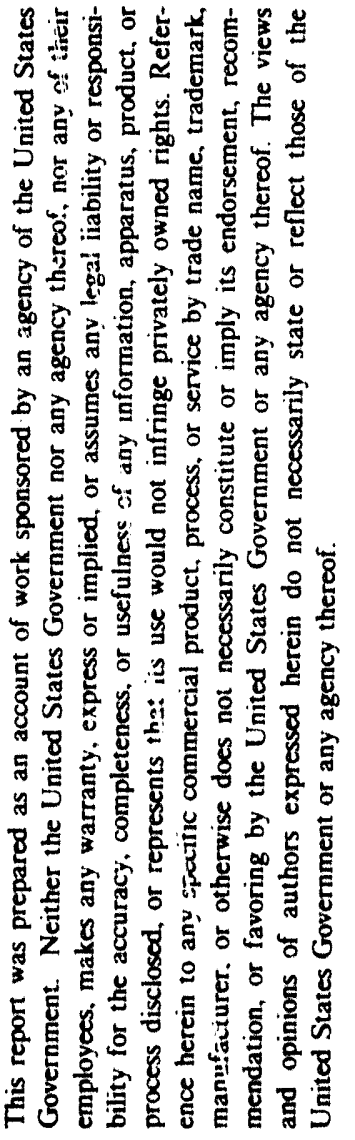

Submitted io:
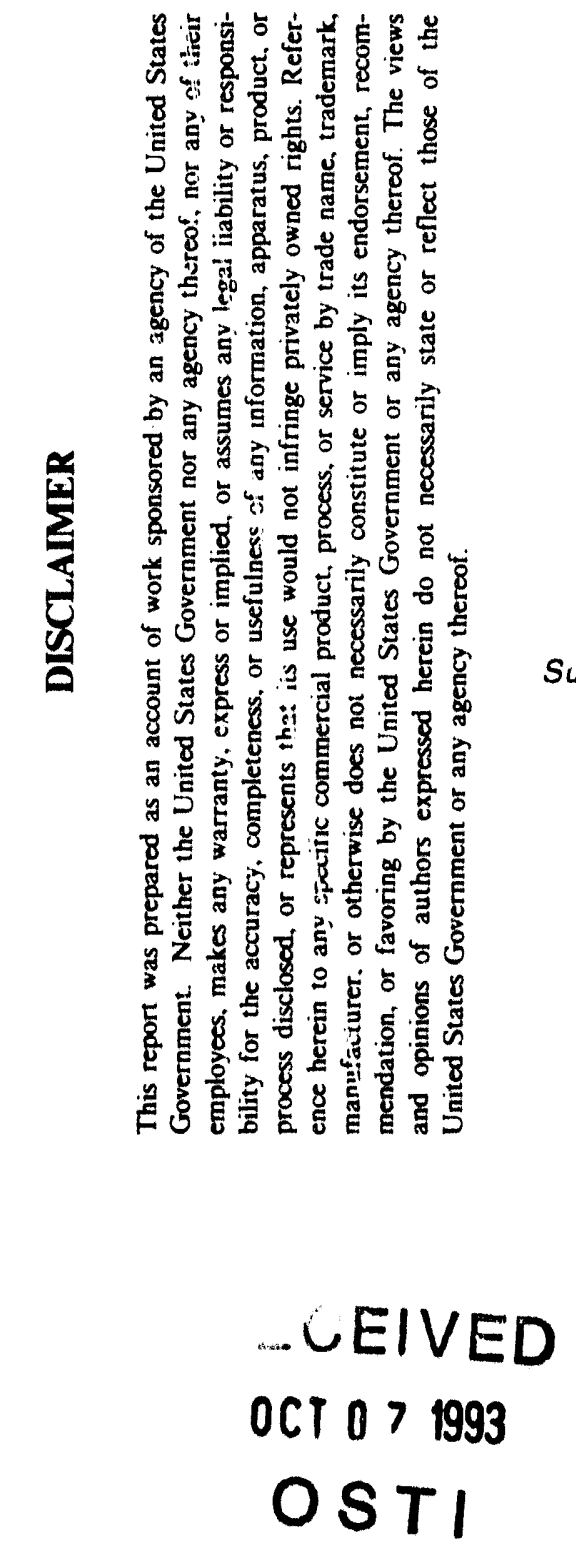

Los Alamos

NATIONAL LABORATORY
DIPOLE-SHEET MULTIPOLE MAGNETS FOR ACCELERATORS.

Peter L. Walstrom

13th International Conference on Magnet Technology
Victoria Conference Centre
Victoria, B.C.
Canada

Soptember 20-24, 1993

MASTER

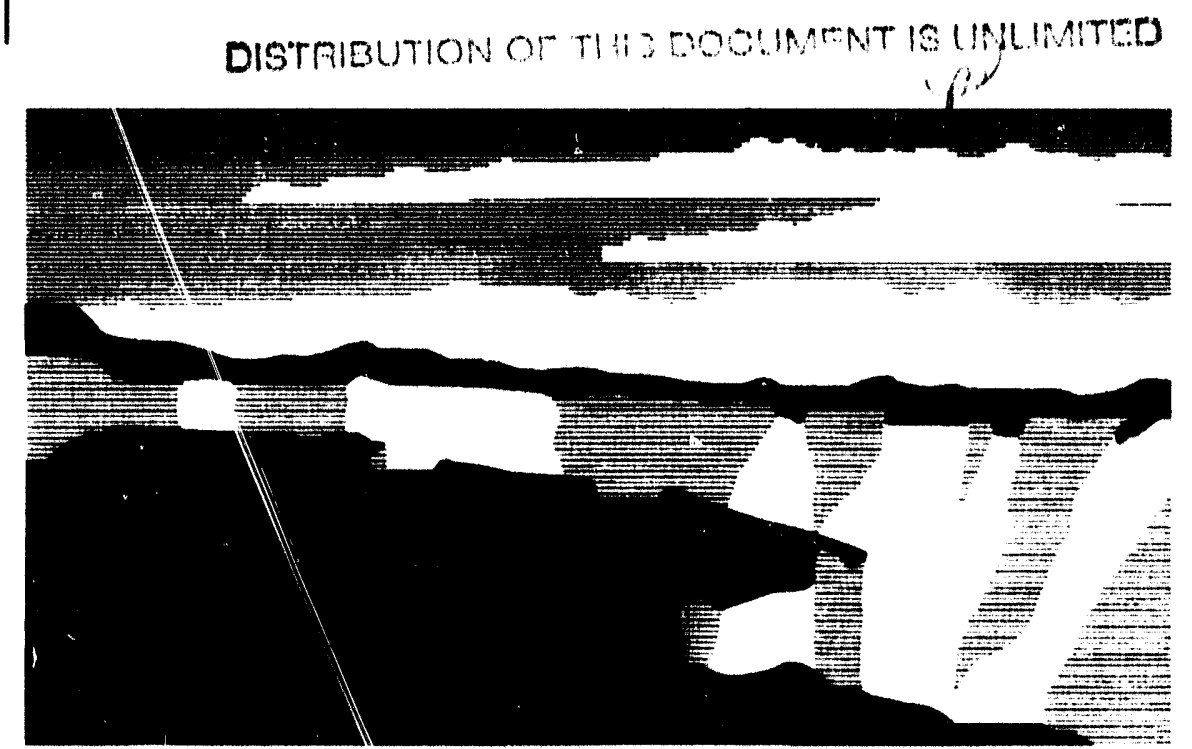

Los Alamos National Laboratory, an aftirmatlve actionequal opportunity employer, is opivrated by the University of California for the U.S. Department of Energy under contract W.7405-ENG-36. By acceptance of this anticle, the publisher recognizes inat the U.S. Government retains a nonexclusive, royalty-Iree llcense lo publish or reproduce the published form of inle contribution, or to allow others to do so, for U.S. Government purposes. The Los Alamos National Laboratory requests that the publisher identify this article as work pertormed under the auspices of the U.S. Department of Energy. 


\title{
Dipole-Sheet Multipole Magnets for Accelerators
}

\author{
P. L. Walstrom \\ Grumman Space and Electronics Systems, Los Alamos National Laboratory, Los Alamos, NM, USA 87545
}

\begin{abstract}
The dipole-sheet formalism can be used to describe both cylindrical current-sheet multipole magnets and cylindrical-bore magnets made up of permanent magnet blocks. For current sheets, the formalism provides a natural way of finding a finite set of turns that approximate a continuous distribution. The formalism is especially useful in accelerator applications where large-bore, short, hlgh-fleld-quallty magnets that are dominated by fringe flelds are needed. A further advantage of the approach is that in systems with either open or cyllndrically symmetric magnetic boundarles, analytical expressions for the three-dimensional fields that are suitable for rapid numerical evaluation can be derived. This development is described in some detall. Alsi), recent developments in higher. order particle-beam optics codes based on the formallsm are described briefly.
\end{abstract}

\section{INTRODUCTION}

High-order optics codes based on realistic threedimensional field models have been developed at Los Alamos II) for the neutral-particle-beam (NPB) program. All of the cases developed to date are in cylindrical coordinates, although many of the ideas can be generalized to other coordinate systems. The computed fields are 3-D solutions of Laplace's equation to machine precision (although only good approximations to the ficlds of real magnets). This is a result of representing the fields in terms of sources, rather than by discrete data on grids, etc. The sources may surround the volume occupied by the magnet, in which case the fields everywhere outside the magnet are represented. In other cases, sources may be placed on the surface of an arbitrary volume that passes through the bore. In this case, fields are represented only within the volume. In many cases of interest in NPB (e.g., large-bore permanent magnet quadrupoles and cylindrical current-sheet magnets such as large-bore steering magnets and printed-circuit-lype correctors), the sources are known a priori. When this is not the case, effective sources can te found by fitling them to the output of finite-element or finite-difference codes, or to experimental data.

\section{INPUT TO BEAM-OPTICS CODES}

Particle-tracking codes require fast 3-D routines for computing the flux-density vector $B$, and sometimes, various derivatives of the vector potential $A$, at arbitrary points within the beam envelope. It is important that the solutions be smoothly varying (differentiable to high order) and preferably exact solutions of Laplace's equation. This rules out most grid-interpolation schemes. For map codes, when the central

Manuscript received September 20, 1993. Work supported and funded by the US Department of Defense, Army Strategic Defense Command, under the auspices of the US Department of Energy. beam trajectory is straight (which is true for most of an NPB device), the fields are represented by generalized on-axis gradients and their $z$ derivatives. (In this paper, $(r, \theta, z)$ and $(\rho, \phi, x)$, respectively, will denote field and source points in cylindrical coordinates). The generalized on-axis gradient is defined as follows: First, the magnetic scalar potential is written in the form

$$
V(r, \theta, z)=\operatorname{Re} \sum_{m=1}^{\infty} e^{i m \theta} F_{m}(r, z)
$$

In (1), real $F_{m}$ correspond to skew $m$-poles and imaginary $F_{m}$ to normal $m$-poles. Also, axisymmetric $(m=0)$ terms are explicitly excluded because they require special treatrient. The mith on-axis generalized gradient is then defined to te

$$
f_{m}(z)=\lim _{r \rightarrow 0} \frac{m F_{m}(r, z)}{r^{m}} \text {. }
$$

The usual power-series form of the scalar potential is written in terms of the $f_{m}$ and their $z$ derivatives as follows:

$$
\begin{aligned}
& V(r, \theta, z)= \\
& \operatorname{Re} \sum_{m=1}^{\infty} e^{i m \theta_{r} m} \sum_{n=0}^{\infty} \frac{(m-1) !}{(n+m) ! n !}\left(-\frac{r^{2}}{4} \frac{d^{2}}{d z^{2}}\right)^{n} f_{m}(z)
\end{aligned}
$$

(For the axisymmetric components, an analogous expression for the vector potential $A_{\phi}$ can be written in terms of $B_{z}$ on axis). If the $f_{m}$ are derived analytically from sources, (3) is valid for $r<r_{\min }$; for field points within the magnet hore, $r_{\min }=a_{\min }$, where $a_{\min }$ is the minimum source radius. For points axially outside the magnet bore, $r_{\min }=\left(a^{2}{ }_{\min }+2^{2}{ }_{\min }\right)^{1 / 2}$, where $z_{\min }$ is the axial distance to the nearest source. However, if the $f_{m}$ are arbitrary functions of $z$ used to fit data, (3) may have much poorer convergence properties and may not correctly represent fields off axis, especially near sources.

\section{THE CYLINDRICAL DIPOLE-SHEET FORMALISM}

Dipole-density distributions can be used to represent both real magnetic material and macroscopic currents $|2|$. Volume currents can be represented by the curl of a fictitious volume dipole distribution $\mathbf{M}$. This formalism automatically gives currents that are divergence-free; moreover, by usc of a gauge transformation, the number of components of $\mathbf{M}$ needed to describe a volume current distribution can be reduced to two. In this paper, only surface-current distributions will be discussed; only one component of $\mathbf{M}$ (the normal component) is needed to describe them. For developable surfaces, including cylinders, this component formally corresponds to the stream function for currents. Streamlines of current 
correspond to contours of constant $\psi$; designs of discrete windings are based on this fact [3]. On the surface of a cylinder, the components of the surface currents are

$$
\begin{aligned}
& j_{\phi}=\frac{\partial \psi(\phi, z)}{\partial z} \\
& j_{z}=-\frac{1}{\rho} \frac{\partial \psi(\phi, z)}{\partial \phi},
\end{aligned}
$$

where $\rho$ is the cylinder radius and $\psi$ the stream function. On disks perpendicular to the $z$ axis, the stream function corresponds to $M_{2}$; currents in this plane contribute nothing to the integrated field strengths, but can be used to represent crror ficlds from leads, etc., and may also be useful for higher-order corrections. Permanent magnet material may in general require three dipole density components for its description. Permanent multipole magnets for accelerators 14], [5] usually have only $\hat{\rho}$ and $\hat{\phi}$ components of $\mathbf{M}$ with $|\mathbf{M}|=M_{0}$, where $M_{0}$ is a constant, and the distribution

$$
\mathbf{M}=M_{0}(\sin m \phi \hat{\rho}-\cos m \phi \hat{\phi})
$$

where $\hat{\rho}$ and $\hat{\phi}$ are unit vectors in the radial and azimuthal directions, respectively. For quadrupoles, $m=2$ in $(5$ ). Expressions with $M_{2}$ are useful for analyzing the effect of unwanted $z$-oriented material in real magnets, and possibly for some corrector elements, although again, z-oriented matcrial contributes nothing to the integrated field strengths.

\section{OPEN.BOUNDARY FIELDS}

In cylindrical coordinates, open-boundary solutions to Laplace's equation can be constructed by superposition of fields from sinusoidal ring sources. The scalar potential for a ring of radius $\rho$, at axial coordinate $x$, with a sinusoidally varying lincar magnetic charge density $\sigma_{m} e^{i m \phi}$, is

$$
\begin{aligned}
& V_{m}(r, \theta, z)= \\
& \frac{\mu_{0} \rho}{2 \pi} \operatorname{Re} \sigma_{m} e^{i m \theta} \sqrt{\frac{1}{\rho r}} Q_{m-1 / 2}\left(\frac{r^{2}+\rho^{2}+(z-x)^{2}}{2 r \rho}\right)
\end{aligned}
$$

where $Q_{m \cdot 1 / 2}$ is a Legendre function of the sccond kind $|6|$. The analogous solutions for sinusoidally-varying linear dipole densities are obtained by differentiation with respect to the source coordinates. For the line dipole density

$$
\mathbf{M}=\left(M_{\rho, m} \hat{\rho}+M_{\phi, m} \hat{\phi}+M_{2, m} \hat{z}\right) e^{i m \phi} \text {, }
$$

on a ring of radius $\rho$ at $x$, the magnetic scalar potential is

$$
\begin{aligned}
& V_{m}(r, \theta, z)=\frac{\mu_{0} \rho}{2 \pi} \operatorname{Re}\left(M_{\rho, m} \frac{\partial}{\partial \rho}-\frac{i m M_{\phi, m}}{\rho}+M_{z, m} \frac{\partial}{\partial x}\right) \\
& e^{i m \theta} \frac{1}{\sqrt{\rho r}} Q_{m-1 / 2}\left(\frac{r^{2}+\rho^{2}+(z-x)^{2}}{2 \rho r}\right) .
\end{aligned}
$$

The expression

$$
M_{\rho, m} \frac{\partial}{\partial \rho}-\frac{i m M_{\phi, m}}{\rho}+M_{z, m} \frac{\partial}{\partial x}
$$

can be regarded as a dipule operator that operates on the elementary ring monopole-source potential (without the factor $\rho$ ) to produce the ring dipole-source potential. This concept anplies not only to the open-boundary solution (6), but to source rings with arbitrary boundaries, provided that all matcrials in the problem are linear. The Legendre function in (6) and (8) can be expressed as a rational function of the complete clliptic integrals $\mathbf{E}$ and $\mathbf{K}$ and their modulus $k$ 171. The modulus $k$ here is given by

$$
k^{2}=\frac{4 \rho r}{(\rho+r)^{2}+(z-x)^{2}}
$$

The on-axis generalized gradient for open boundaries is oblained from (8) by first expressing $Q$ as a hypergenmetric series, and then taking the limit defined by (2). The result is

$$
\begin{aligned}
& f_{m}(z)=\frac{(2 m-1) ! ! \mu_{0} \rho}{2^{m+1}(m-1) !}\left(M_{\rho, m} \frac{\partial}{\partial \rho}-M_{\phi, m} \frac{i m}{\rho}+M_{z, m} \frac{\partial}{\partial x}\right) \\
& {\left[\rho^{2}+(z-x)^{2}\right]^{m+1 / 2}}
\end{aligned}
$$

Current shects on cylinders can be built up by superposition of terins of the form $\psi_{m}(\phi, x)=w_{m}(x) e^{i m \phi}$. Here, $w_{m}$ is called a shape function. For current sheets on the $z$ interval $[-l,+1], M_{\rho, m}$ is the only term in (11), and is replaced by $w_{m}(x) d x ;(11)$ is then integrated in $x$ as follows:

$$
\begin{aligned}
& f_{m}(z)= \\
& \frac{(2 m-1) ! ! \mu_{0} \rho}{2^{m+1}(m-1) !} \int_{-1}^{+1} w_{m}(x) \frac{\partial}{\partial \rho}\left[\frac{\rho^{m}}{\left[\rho^{2}+(z-x)^{2}\right]^{m+1 / 2}}\right] d x .
\end{aligned}
$$

For permanent multipole magnets of rectangular cross-scition in the $r$-z plane, with inne dius $a_{1}$, outer radius $a_{2}$, and the dipole-density distribution of (5), the expression for $f_{m}$ is

$$
\begin{aligned}
& f_{m}(z)= \\
& -\frac{(2 m+1) ! ! \mu_{0} M_{0}}{2^{m+1}(m-1) !} \int_{-1}^{+1} \int_{a_{1}}^{a_{2}} \frac{\rho^{m+2} d \rho d x}{\left[\rho^{2}+(z-x)^{2}\right]^{m+3 / 2}} .
\end{aligned}
$$

For many functional forms of $w_{m}(x)$, including piecewisecontinuous polynomials, the integrals of (12) needed for $f_{m}$ and its derivatives can be done in closed form. The double integrals of (13) for $f_{m}$ and its derivatives can also be done in closed form. Fast routines for evaluating the integrals of (12) with a variety of shape functions, and the double integrals in 
(13) have been incorporated in the higher-order optics codes MAR YLIE and TLIE [1].

For particle tracking, one wants fast algorithms for computing $\mathbf{B}$, and sometimes $\mathbf{A}$ and certain of its derivatives. This is complicated bv the fact that $(8)$ is not easily integrated over $x$ in closed form, especially with shape functions inside the integral sign. For ficld points with radius $r<1 / 2 r_{\mathrm{min}}$, where $r_{\text {min }}$ is the radius of convergence of (3), the most efficient scheme is to use for $\mathbf{B}$ the gradient of (3). The onaxis gradients $f_{m}$ and their derivatives are computed by use of (12) or (13). The vector potential A can also easily be expressed in terms of the $f_{m}$ and their $z$ derivatives. Enough terms are used to ensure the desired accuracy. Points with larger radii require use of a different approach. If the source is a line source (i.e., of no extent in $z$ or $\rho$ ), the cllipticintegral form of $Q_{m \cdot 1 / 2}$ is used. For distributed sources on the interval $[-1,+1]$ on a cylinder of radius $\rho, \partial / \partial x$ is replaced by - $\partial / \partial_{z}$; this is allowed here by translational symmetry in 2 . The following angular-integral form of (8) is then used $[6]$ :

$$
\begin{aligned}
& V_{m}(r, \theta, z)=\frac{\mu_{0} \rho}{\pi} \operatorname{Re} e^{i m \theta} \\
& \int_{0}^{\pi / 2} \int_{-1}^{+1}\left(M_{\rho, m}(x) \frac{\partial}{\partial \rho}-\frac{i m M_{\phi, m}(x)}{\rho}-M_{2, m}(x) \frac{\partial}{\partial z}\right) \\
& \frac{(-1)^{m} \cos 2 m \alpha d \alpha d x}{\sqrt{(\rho+r)^{2}+(z-x)^{2}-4 \rho r \sin ^{2} \alpha}} .
\end{aligned}
$$

The $x$ integration is performed first. For current sheets, $M_{\rho, m}(x)$ is the shape-function part of the stream function., and is represented by a piecewise-continuous polynomial. For permanent multipole magnets, $M_{\rho, m}=-i M_{0}, M_{\phi, m}=\cdot M_{0}$, and $M_{2, m}=0$. In both cases, the $x$ integrals are of the form

$$
S_{n}=\int \frac{x^{n} d x}{\sqrt{(\rho+r)^{2}+(z-x)^{2}-4 \rho r \sin ^{2} \alpha}} \text {. }
$$

$n=(), 1,2, \cdots$. Closed-form expressions can he found for all of the $S_{n}$. The next step in finding $B$ is to move both the dipole operator and the gradient operator inside the integral sign for the angular integration and apply them to the $S_{n}$. Each component of B becomes a finite sum of angular integrals on $(0, \pi / 2)$. In some cases, these integrals can be expressed in terms of the complete elliptic integrals $\mathbf{E}, \mathbf{K}$, and $\Pi$, for which fast numerical evaluation algorithms hased on the Landen transformation are available $|8|$. When this is not possible, direct Landen numerical quadrature can always he used $|9|$. Landen quadrature applies to integrals of the form

$$
T=\int_{0}^{\pi / 2} \frac{F(R)}{R} d x .
$$

where $R=\left(\mu^{2} \cos ^{2} \alpha+v^{2} \sin ^{2} \alpha\right)^{1 / 2}$, and $F$ is any analytic function on $\{0, \pi / 2\}$. Landen quadrature converges exponentially in the number of function evaluations.

\section{OTHER BOUNDARY CONDITIONS}

The infinite-permeablility-iron approximation will be used here. If all iron components are axisymmetric, the only $m$ values in the scalar potential will be those in the fourier decomposition of the sources. The simplest axisymmetric boundary-value problem of interest to designers of accelerator magnets is an infinite circular cylinder with sources inside. (Finite-length boundaries would require a boundary-integral approach, and are not treated here. However, some of the results of this paper should be useful for evaluating boundary integrals). The case of a dipole. density source distribution on the surface of a cylinder coaxial with a boundary of radius $b$ will be treated below (this includes surface currents). As in the open-boundary casc, the sources are first decomposed into a Fourier series in angle. The Fourier transform in $x$ of the mth Fourier angular component of the dipole density is next defined to be

$$
\begin{aligned}
& \mu_{\rho, m}(s) \hat{\rho}+\mu_{\phi, m}(s) \dot{\phi}+\mu_{z, m}(s)= \\
& \frac{1}{2 \pi} \int_{-\infty}^{+\infty}\left|M_{\rho, m}(x) \hat{\rho}+M_{\phi, m}(x) \dot{\phi}+M_{z, m}(x) \hat{z}\right| e^{L s x} d x .
\end{aligned}
$$

Analytic forms for these transforms are available for most cases of interest. For $r<\rho$, the Fouricr-integral expression for the mulh Fourier series component of the scalar potential is

$$
\begin{aligned}
& V_{m}(r, \theta, z)=\mu_{0} \operatorname{Re} e^{u m \theta} \\
& \int_{-\infty}^{+\infty}\left[\mu_{\rho, m}(s) \rho \frac{\partial}{\partial \rho}-i m \mu_{\phi, m}(s)-i s \mu_{z, m}(s)\right] \\
& I_{m}(s r)\left[K_{m}(s \rho)-I_{m}(s \rho) \frac{K_{m}(s b)}{I_{m}(s b)}\right] e^{L i z} d s,
\end{aligned}
$$

where $I_{m}$ and $K_{m}$ are modified Bessel functions. For $r>\rho, r$ and $\rho$ are exchanged in the Bessel-function arguments in (18). Fourier-integral expressions for the field compronents are derived from (18) by applying the gradient operator and can be evaluated numerically by use of Filon quadrature. This procedure is not very fast in comparison to other methods discussed here, but it is useful for checking the laster algorithms, since (18) applies not only to cylindrical boundaries, but also 10 open boundaries when the $1 \mathrm{~cm}$ containing $b$ is dropped. The on-axis generalized gradient (sec (1) and (2)) is easily oblained from (18) by using the serics expansion for $I_{m}$ and laking the limit according $(0)(2)$ :

$$
\begin{aligned}
& f_{m}(z)= \\
& \frac{\mu_{0}}{(m-1) ! 2^{m}} \int_{m \infty \infty}^{+\infty}\left[\mu_{p, m}(s) \rho \frac{\partial}{\partial \rho}-i m \mu_{\phi, m}(s)-i s \mu_{2, m}(s)\right] \\
& s^{\prime m}\left[K_{m}(s \rho)-I_{m}(s \rho) \frac{K_{m}(s b)}{I_{m}(s b)}\right] e^{\omega s z} d s .
\end{aligned}
$$

The ahove Fourier-integral expressions are also useful for linding expressions for the $z$ integrals of ficlds and gradients, since integration of $e^{i s z}$ over $[-\infty,+\infty)$ gives a della functuon in s. The z-integrated scalar potential is found to be 


$$
\begin{aligned}
& \int_{-\infty}^{+\infty} V_{m}(r, \theta, z) d z=-\frac{\mu_{0}}{2} \operatorname{Re} e^{i m \theta}\left(\frac{r}{\rho}\right)^{m} \\
& \int_{-\infty}^{+\infty}\left\{\left[1+\left(\frac{\rho}{b}\right)^{2 m}\right] M_{\rho, m}(x)+i\left[1-\left(\frac{\rho}{b}\right)^{2 m}\right] M_{\phi, m}(x)\right\} d x
\end{aligned}
$$

The $z$-integrated generalized on-axis gradient is

$$
\begin{aligned}
& \int_{-\infty}^{+\infty} f_{m}(z) d z=-\frac{\mu_{0} m}{2 \rho^{m}} \\
& \int_{-\infty}^{+\infty}\left\{\left[1+\left(\frac{\rho}{b}\right)^{2 m}\right] M_{\rho, m}(x)+i\left[1-\left(\frac{\rho}{b}\right)^{2 m}\right] M_{\phi, m}(x)\right\} d x .
\end{aligned}
$$

The axial component of the dipole density makes no contribution to the integrated scalar potential or gradient if the distribution is bounded in $z$ (as was assumed in deriving (20)).

For faster field calculation with infinite-cylinder boundarics, the Bessel-function serirs form of (18) is used:

$$
\begin{aligned}
& V_{m}(r, \theta, z)=\mu_{0} \rho \operatorname{Re} e^{i m \theta} \sum_{n=1}^{\infty} \frac{J_{m}\left(k_{n m} r\right)}{k_{n m} b^{2} J_{m+1}^{2}\left(k_{n m} b\right)} \\
& \int_{-1}^{+1}\left[M_{\rho, m}(x) \frac{\partial}{\partial \rho}-\frac{i m M_{\phi, m}(x)}{\rho}-M_{z, m} \frac{\partial}{\partial z}\right] \\
& J_{m}\left(k_{n m} \rho\right) e^{-k_{m}|z-x|} d x,
\end{aligned}
$$

where $k_{n m}$ is the $n$th root of the equation $J_{m}\left(k_{n m} b\right)=0$. The above integral must be performed variously, according to whether $|z|>1$ or $|z|<l$. For points with $|z|<l$, the integral gives constants in $z$ times positive exponentials in $z$ when $z<0$, and negative exponentials when $2>0$. For interior points, the integrals yield sums of functions of $z$ times positive and negative exponentials in 7. However, for field points with 2 near $\pm l$, the convergence of the series is slow. For these points, a sum of two series solutions on a finite interval $[-D,+D]$ around $[-1,+1]$, is used $[3]$. The first series is

$$
\begin{aligned}
& V_{m, 1}(r, \theta, z)=\mu_{0} \rho \operatorname{Rc} e^{i m \theta} \sum_{k=1}^{\infty} I_{m}\left(\lambda_{k} r\right) \\
& {\left[C_{m, k}^{\rho} \frac{\partial}{\partial \rho}-\frac{i m}{\rho} C_{m, k}^{\dagger}-C_{m, k}^{z} \frac{\partial}{\partial z}\right]} \\
& {\left[K_{m}\left(\lambda_{k} \rho\right)-I_{m}\left(\lambda_{k} \rho\right) \frac{K_{m}\left(\lambda_{k} b\right)}{I_{m}\left(\lambda_{k} b\right)}\right] \cos \lambda_{k},}
\end{aligned}
$$

where $\lambda_{k}=(2 k-1) \pi / 2 D$ and the Fourier coeffricients are defined by the equations

$$
C_{m, k}^{\rho}=\frac{1}{D} \int_{-D}^{+D} M_{\rho, m}(x) \cos \lambda_{k} x d x .
$$

etc. for the parts of the dipole density components that are even in $x$. Similar expressions containing sines can be written for the components that are odd in $x$. A second series is added to $V_{1}$ to correct for the fact that the actual potential is not zero for $z= \pm D$. For sources even in $z$, the series is

$V_{2}(r, \theta, z)=\mu_{0} \operatorname{Re} e^{i m \theta} \sum_{n=1}^{\infty} q_{n m} e^{-k_{n m} D}\left(\frac{\cosh k_{n m} z}{\cosh k_{n m} D}\right) \gamma_{m}\left(k_{n m r}\right)$

where the $q_{n m}$ are given by the expression

$$
\begin{aligned}
& q_{n m}=\frac{1}{k_{n m} b^{2} J_{m+1}^{2}\left(k_{n m} b\right)} \\
& \int_{-1}^{+1}\left[M_{\rho, m}(x) \frac{\partial}{\partial \rho}-\frac{i m}{\rho} M_{\phi, m}(x)-M_{2, m}(x) \frac{\partial}{\partial z}\right] \\
& J_{m}\left(k_{n m} \rho\right) e^{k_{m m} x} d x .
\end{aligned}
$$

\section{REFERENCES}

1. P. L. Walstrom, F. Neri, and C.T. Mottershead, "HighOrder Optics of Multipole Magnets", paper given at the 1990 Lincar Accelcrator Conference, Albuquerque, N.M., Scpt. 10. 14. Proceedings available from Los Alamos National Laboratory as LA-12(0)4-C, March, $1(20) 1$.

2. P. L. Walstrom, "Magnetic Ficlds from Distributions of Dipoles on Cylindrical Surfaces", Los Alamos National Laboratory internal report LA.UR-91.3496, Nov. 1991.

3. P. L. Walstrom, "Magnetic Ficlds and Inductances of Cylindrical Current Sheet Magnets", Los Alamos National Laboratory internal report L.A-UR-90-4090, Dec. 199().

4. A. N. Gerbert, S.B. Mukho, Ya. D. Rabinovich, and V. S. Skachkov, "Quadrupole Lens with Poles of Implicit Form based on Permanent Magnets", Pribory $i$ Tekhnika Eksperimenta, no. 1, pp. 49-51, Jan.-Feb. 1980).

5. K. Halbach, "Strong Rare Earth Cobalt Quadrupoles", Proc. 1979 IEEE Particle Accelerator Conference. NS.26 3882 (1979).

6. C. Snow, Hypergeometric and Legendre Functions with Applications to Integral Equations of Potential Theory, published as U.S. Department of Commerce, National Burcau of Standards Applied Mathematics Series 19, May, 1952.

7. Handbook of Mathematical Functions, ed. by $M$. Abramowitz and I. Stegun, Dover Publications, Inc. , New York, NY, 1972 (republication of the National Burcau of Standards handbook of 1964 with the same tille). The expression is derived by symbolic recursion with 8.5.3, staring with 8.13.3 and 8.13.7. Numerical recursion can be unstable and should be avoided.

8. W. H. Press, B. P. Flannery, S A. Teukolsky, and W. T. Fetcerling, Numerical Recipes, Cambridge University Press, NY, 1989.

9. W. Bartky, "Nunierical Calculation of a Generalized Completc Elliptic Integral", Reviews of Modern Physics 10, 264 (1938). 

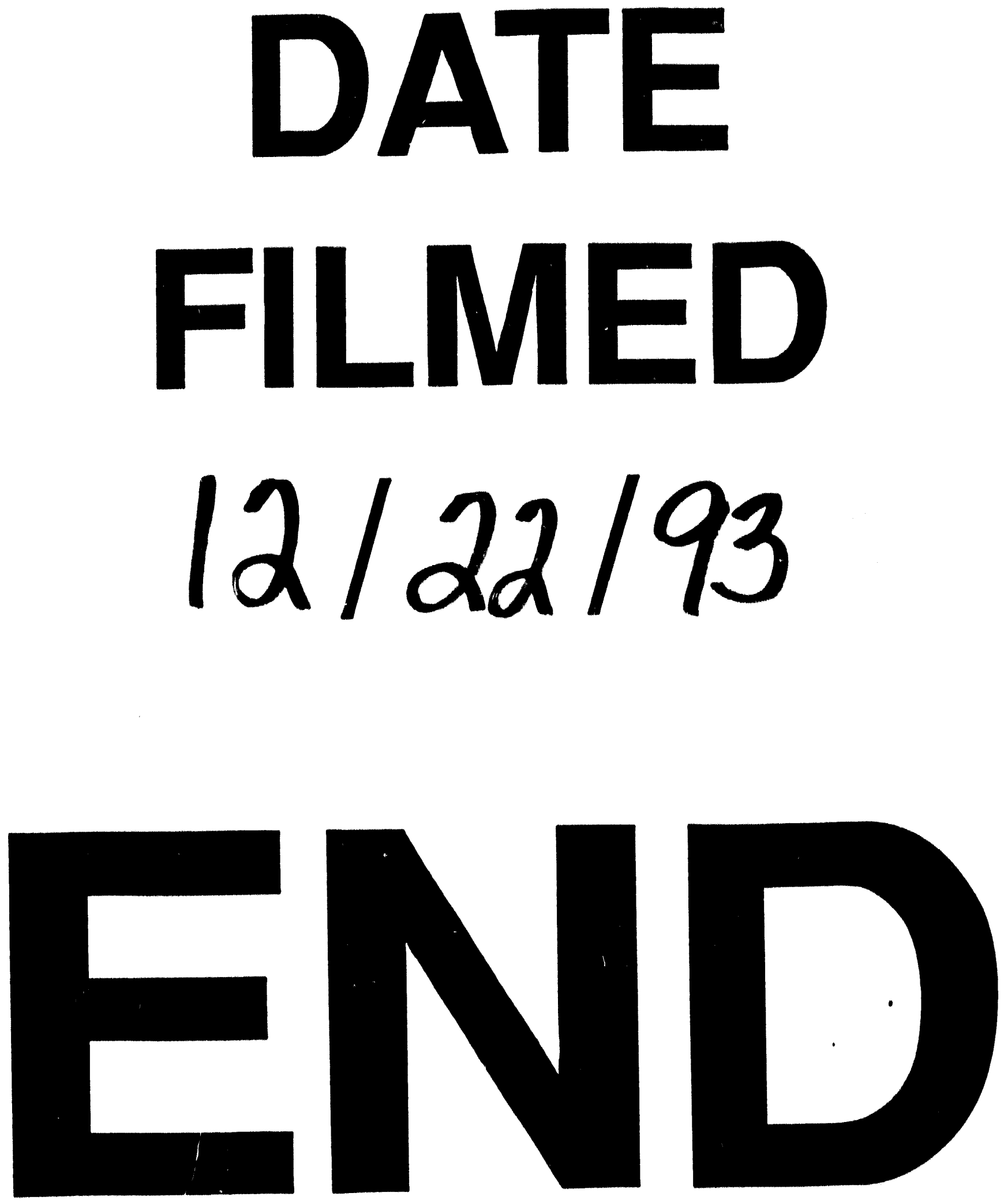
